\title{
Sufficient conditions for regular solvability of an arbitrary order operator-differential equation with initial-boundary conditions
}

\author{
Nashat Faried ${ }^{1}$, Abdel Baset I. Ahmed ${ }^{2 *}$ and Mohamed A. Labeeb ${ }^{3}$
}

"Correspondence:

abdel2007@yandex.ru

${ }^{2}$ Helwan University, Cairo, Egypt

Full list of author information is

available at the end of the article

\section{Springer}

\begin{abstract}
On this paper, for an arbitrary order operator-differential equation with the weight $e^{\frac{-\alpha t}{2}}, \alpha \in(-\infty,+\infty)$, in the space $W_{2}^{n+m}\left(R_{+} ; H\right)$, we attain sufficient conditions for the well-posedness of a regular solvable of the boundary value problem. These conditions are provided only by the operator coefficients of the investigated equation where the leading part of the equation has multiple characteristics. We prove the connection between the lower bound of the spectrum of the higher-order differential operator in the main part and the exponential weight and also obtain estimations of the norms of operator intermediate derivatives. We apply the results of this paper to a mixed problem for higher-order partial differential equations (HOPDs).

MSC: 34A12; 34G10; 34k10; 35J40; 47D03

Keywords: Initial-boundary value problems (IBVPS); The spectral resolution; A positive-definite operator; Bounded operator; Hilbert space; Integral operator: Regular solvability
\end{abstract}

\section{Introduction}

The theory of initial-boundary value problems of operator-differential equations in a Banach or Hilbert space is of great value and secures the possibility of looking at ordinary and partial differential operators [27]. It is worth mentioning that the principal parts of the investigated equations have multiple characteristics and thus appear in applications, for instance, by modeling the stability of the plates from the plastic and in particular, in the dynamics problems of arches and rings [25,26]. The solvability of initial boundary value problems for higher-order operator-differential equations has been researched by many authors, for example, A. A. Gasymov, S. S. Mirzoev, V. I. Gorbachuk, M. L. Gorbachuk, S. Yakubov, V. N. Pilipchuk, and their followers [7, 11, 22-25, 27].

In a separable Hilbert space $\mathrm{H}$, we study the following initial-boundary value problem:

$$
\begin{aligned}
& \prod_{k=1}^{m}\left(-\frac{d}{d t}+r_{k} A\right) \prod_{k=1}^{n}\left(\frac{d}{d t}+r_{k} A\right) u(t)+\sum_{j=1}^{n+m} A_{j} \frac{d^{n+m-j}}{d t^{n+m-j}} u(t)=f(t), \\
& t \in R_{+}=[0, \infty)
\end{aligned}
$$

(c) The Author(s) 2020. This article is licensed under a Creative Commons Attribution 4.0 International License, which permits use, sharing, adaptation, distribution and reproduction in any medium or format, as long as you give appropriate credit to the original author(s) and the source, provide a link to the Creative Commons licence, and indicate if changes were made. The images or other third party material in this article are included in the article's Creative Commons licence, unless indicated otherwise in a credit line to the material. If material is not included in the article's Creative Commons licence and your intended use is not permitted by statutory regulation or exceeds the permitted use, you will need to obtain permission directly from the copyright holder. To view a copy of this licence, visit http://creativecommons.org/licenses/by/4.0/. 


$$
\frac{d^{s} u(0)}{d t^{s}}=0, \quad s=\overline{0, n+m-2},
$$

where $A$ is a self-adjoint positively defined operator, $\left(A=A^{*} \geq \gamma_{0} E, \gamma_{0}>0\right), \gamma_{0}$ is the lower bound of spectrum $\left(\gamma_{0} \in \sigma(A)\right), r_{k} \neq 0, r_{m}=r_{n}=1$ for all $r_{m}, r_{n}$, and $A_{j}, j=\overline{1, n+m}$, are linear unbounded operators. All derivatives here are perceived in the sense of distributions theory.

$$
\text { Let } f(t) \in L_{2, \alpha}\left(R_{+} ; H\right), u(t) \in W_{2, \alpha}^{n+m}\left(R_{+} ; H\right) \text {, and } \alpha \in R \text {, where }
$$

$$
L_{2, \alpha}\left(R_{+} ; H\right)=\left\{f(t):\|f(t)\|_{L_{2, \alpha}\left(R_{+} ; H\right)}=\left(\int_{0}^{+\infty}\|f(t)\|_{H}^{2} e^{-\alpha t} d t\right)^{\frac{1}{2}}<+\infty\right\}
$$

and

$$
W_{2, \alpha}^{n+m}\left(R_{+} ; H\right)=\left\{u(t): \frac{d^{n+m} u(t)}{d t^{n+m}} \in L_{2, \alpha}\left(R_{+} ; H\right), A^{n+m} u(t) \in L_{2, \alpha}\left(R_{+} ; H\right)\right\}
$$

with the norm $[14,15]$

$$
\|u\|_{W_{2, \alpha}^{n+m}\left(R_{+} ; H\right)}=\left(\left\|A^{n+m}(u)\right\|_{L_{2, \alpha}\left(R_{+} ; H\right)}^{2}+\left\|\frac{d^{n+m}(u)}{d t^{n+m}}\right\|_{L_{2, \alpha}\left(R_{+} ; H\right)}^{2}\right)^{\frac{1}{2}} .
$$

At $\alpha=0$, for simplification, we denote the space $L_{2,0}\left(R_{+} ; H\right)$ by $L_{2}\left(R_{+} ; H\right)$ and the space $W_{2,0}^{n+m}\left(R_{+} ; H\right)$ by $W_{2}^{n+m}\left(R_{+} ; H\right)[4-9]$.

Definition 1 If for any $f(t) \in L_{2, \alpha}\left(R_{+} ; H\right)$, there exists a vector function $u(t) \in W_{2, \alpha}^{n+m}\left(R_{+} ; H\right)$ that satisfies (1) almost everywhere in $R_{+}$, then it is called a regular solution of (1).

Definition 2 If for any function $f(t) \in L_{2, \alpha}\left(R_{+} ; H\right)$, there exists a regular solution of (1) satisfying the boundary conditions (2) in the sense that [24]

$$
\lim _{t \rightarrow 0}\left\|A^{(n+m)-i-\frac{1}{2}} \frac{d^{i} u(t)}{d t^{i}}\right\|_{H}=0, \quad i=\overline{0, n+m-2},
$$

then

$$
\|u\|_{W_{2, \alpha}^{n+m}\left(R_{+} ; H\right)} \leq \mathrm{const}\|f\|_{L_{2, \alpha}\left(R_{+} ; H\right)} .
$$

The studies of the last 60 years have enhanced the theory of operator-differential equations with considerable results. The theory of initial-value problems of operator-differential equations in a Banach or Hilbert space is useful because it facilitates studying equations of parabolic and elliptic differential operators with initial-boundary conditions, possibly, looking at ordinary and partial differential operators. Nowadays many papers concerning the study of initial value problems of the operator-differential equations in Banach spaces have been published. In both semiaxis and finite interval, second-order operatordifferential equations with zero weight exponential are studied [3, 5, 6, 23, 24].

Gasymov [8-10] analyzed both the solvability of operator-differential equations and the multiple completeness of some eigen- and associated vectors of corresponding operator pencils. His works are the most valuable as they motivated many papers, including the 
present one and others to be mentioned further. Moreover, the solvability of operatordifferential equations in Hilbert spaces with exponential weight has been extensively studied. Second-, third-, and fourth-order operator-differential equations with multiple characteristics with exponential weight have been studied on the semiaxis and the whole axis $[2,22]$. Moreover, general higher-order operator-differential equations with multiple characteristic in a Sobolev-type space with exponential weight have not been studied yet. In the present paper, we formulate sufficient conditions for the initial-boundary value problem to be regularly solvable.

\section{Main results}

From the theorem on intermediate derivatives $[7,19]$ we have that if $u(t) \in W_{2, \alpha}^{n+m}\left(R_{+} ; H\right)$, then

$$
A^{j} \frac{d^{n+m-j} u(t)}{d t^{n+m-j}}: W_{2, \alpha}^{n+m}\left(R_{+} ; H\right) \rightarrow L_{2, \alpha}\left(R_{+} ; H\right) \rightarrow L_{2, \alpha}\left(R_{+} ; H\right), \quad j=\overline{1, n+m},
$$

and the following inequalities are valid:

$$
\left\|A^{j} \frac{d^{n+m-j} u(t)}{d t^{n+m-j}}\right\|_{L_{2, \alpha}\left(R_{+} ; H\right)} \leq c_{j}\|u\|_{W_{2, \alpha}^{n+m}\left(R_{+} ; H\right)}, \quad j=\overline{1, n+m}
$$

In the present paper, for any natural number $n, r_{1}=r_{2}=r_{3}=\cdots=r_{n}=1$, and $m=1$, we obtain

$$
\begin{aligned}
& \left(-\frac{d}{d t}+A\right) \prod_{k=1}^{n}\left(\frac{d}{d t}+r_{k} A\right) u(t)+\sum_{j=1}^{n+1} A_{j} \frac{d^{n+1-j}}{d t^{n+1-j}} u(t)=f(t), \\
& \frac{d^{s} u(0)}{d t^{s}}=0, \quad s=\overline{0, n-1} .
\end{aligned}
$$

Equation (4) can be formulated in the form

$$
P u(t) \equiv P_{0} u(t)+P_{1} u(t)=f(t)
$$

where

$$
\begin{aligned}
& P_{0} u=\left(-\frac{d}{d t}+A\right)\left(\frac{d}{d t}+A\right)^{n} u, \\
& P_{1} u=\sum_{j=1}^{n+1} A_{j} \frac{d^{n+1-j}}{d t^{n+1-j}} u,
\end{aligned}
$$

and $f(t) \in L_{2, \alpha}\left(R_{+} ; H\right), u(t) \in W_{2, \alpha}^{n+1}\left(R_{+} ; H\right)$.

Theorem 3 Let $A=A^{*} \geq \gamma_{0} E\left(\gamma_{0}>0, E\right.$ is the unit operator $)$ and $|\alpha|<2 \gamma_{0}$. Then the operator $P_{0}$ is an isomorphism between the spaces $W_{2, \alpha}^{n+1}\left(R_{+} ; H\right)$ and $L_{2, \alpha}\left(R_{+} ; H\right)$.

Proof Let $u(t)=v(t) e^{\frac{\alpha}{2} t}$, then we get the problem $P_{0, \alpha} v(t)=g(t)$, where $v(t) \in W_{2}^{n+1}\left(R_{+} ; H\right)$, $g(t)=f(t) e^{\frac{\alpha}{2} t} \in L_{2}\left(R_{+} ; H\right)$ with

$$
P_{0, \alpha} v(t)=\left(-\left(\frac{d}{d t}+\frac{\alpha}{2}\right)+A\right)\left(\frac{d}{d t}+\frac{\alpha}{2}+A\right)^{n} v(t)=g(t) .
$$


Fried et al. Advances in Difference Equations

(2020) 2020:104

Page 4 of 14

Since the mapping $v(t) \rightarrow u(t) e^{-\frac{\alpha}{2} t}$ is an isomorphism between the spaces $W_{2}^{n+1}\left(R_{+} ; H\right)$ and $W_{2, \alpha}^{n+1}\left(R_{+} ; H\right)$, it is sufficient to prove that $P_{0, \alpha}: W_{2}^{n+1}\left(R_{+} ; H\right) \rightarrow L_{2}\left(R_{+} ; H\right)$ is an isomerphism [12, 13], so we must find the solution of (6) in the form

$$
v(t)=v_{1}(t)+v_{0}(t)=\int_{0}^{+\infty} G(t-s) g(s) d s+v_{0}(t)
$$

as follows.

Using the Fourier transforms for the equation $P_{0, \alpha} v(t)=g(t)$, we obtain

$$
\left(-i \xi E-\frac{\alpha}{2} E+A\right)\left(i \xi E+\frac{\alpha}{2} E+A\right)^{n} \tilde{v}(\xi)=\tilde{g}(\xi), \quad \xi \in R_{+},
$$

where $\tilde{v}(\xi), \tilde{g}(\xi)$ are the Fourier transforms of the functions $v(t), g(t)$, respectively, and moreover

$$
\tilde{v}(\xi)=\left(-i \xi E-\frac{\alpha}{2} E+A\right)^{-1}\left(i \xi E+\frac{\alpha}{2} E+A\right)^{-n} \tilde{g}(\xi) .
$$

Hence

$$
\begin{aligned}
v_{1}(t) & =\frac{1}{\sqrt{2 \pi}} \int_{0}^{+\infty}\left(-i \xi E-\frac{\alpha}{2} E+A\right)^{-1}\left(i \xi E+\frac{\alpha}{2} E+A\right)^{-n} \tilde{g}(\xi) e^{i \xi t} d \xi \\
& =\frac{1}{2 \pi} \int_{0}^{+\infty}\left(-i \xi E-\frac{\alpha}{2} E+A\right)^{-1}\left(i \xi E+\frac{\alpha}{2} E+A\right)^{-n}\left(\int_{0}^{+\infty} g(s) e^{-i \xi s} d s\right) e^{i \xi t} d \xi \\
& =\int_{0}^{+\infty}\left(\frac{1}{2 \pi} \int_{0}^{+\infty}\left(-i \xi E-\frac{\alpha}{2} E+A\right)^{-1}\left(i \xi E+\frac{\alpha}{2} E+A\right)^{-n} e^{i \xi(t-s)} d \xi\right) g(s) d s \\
& =\int_{0}^{+\infty} G(t-s) g(s) d s .
\end{aligned}
$$

Then the integral operator is

$$
G(t-s)=\frac{1}{2 \pi} \int_{0}^{+\infty}\left(-i \xi E-\frac{\alpha}{2} E+A\right)^{-1}\left(i \xi E+\frac{\alpha}{2} E+A\right)^{-n} e^{i \xi(t-s)} d \xi .
$$

Taking $i \xi=\omega$, we have

$$
G(t-s)=\frac{1}{2 \pi i} \int_{-i \infty}^{+i \infty}\left(-\omega E-\frac{\alpha}{2} E+A\right)^{-1}\left(\omega E+\frac{\alpha}{2} E+A\right)^{-n} e^{\omega(t-s)} d \omega .
$$

If $\mu \in \sigma(A)$, then

$$
G(t-s)=\frac{1}{2 \pi i} \int_{-i \infty}^{+i \infty} \frac{e^{\omega(t-s)}}{\left(-\omega-\frac{\alpha}{2}+\mu\right)\left(\omega+\frac{\alpha}{2}+\mu\right)^{n}} d \omega
$$

and for $t>s$, we get

$$
G(t-s)=\operatorname{Re} s_{\omega=-\frac{\alpha}{2}-\mu} \frac{e^{\omega(t-s)}}{\left(-\omega-\frac{\alpha}{2}+\mu\right)\left(\omega+\frac{\alpha}{2}+\mu\right)^{n}}
$$




$$
\begin{aligned}
& =\left(\frac{1}{(n-1) !}\right) \lim _{\omega \rightarrow-\frac{\alpha}{2}-\mu} \frac{d^{n-1}}{d \omega^{n-1}}\left[\frac{e^{\omega(t-s)}}{\left(-\omega-\frac{\alpha}{2}+\mu\right)}\right] \\
& =\left(\frac{1}{(n-1) !}\right) \sum_{k=0}^{n-1} \frac{2^{(-n)}(n-1) ![\mu(t-s)]^{k}}{2^{(-k)} k !} e^{-\left(\mu+\frac{\alpha}{2}\right)(t-s)} \mu^{-n} \\
& =\sum_{k=0}^{n-1} \frac{2^{(-n)}[\mu(t-s)]^{k}}{2^{(-k)} k !} e^{-\left(\mu+\frac{\alpha}{2}\right)(t-s)} \mu^{-n}
\end{aligned}
$$

Similarly, for $t<s$, we have

$$
\begin{aligned}
G(t-s) & =\frac{1}{2 \pi i} \int_{-i \infty}^{+i \infty} \frac{e^{\omega(t-s)}}{\left(-\omega-\frac{\alpha}{2}+\mu\right)\left(\omega+\frac{\alpha}{2}+\mu\right)^{n}} d \omega \\
& =\operatorname{Re} s_{\omega=\mu-\frac{\alpha}{2}} \frac{e^{\omega(t-s)}}{\left(-\omega-\frac{\alpha}{2}+\mu\right)\left(\omega+\frac{\alpha}{2}+\mu\right)^{n}} \\
& =\lim _{\omega \rightarrow \mu-\frac{\alpha}{2}}\left[\frac{e^{\omega(t-s)}}{\left(\omega+\frac{\alpha}{2}+\mu\right)^{n}}\right] \\
& =(2)^{-n} e^{\left(\mu-\frac{\alpha}{2}\right)(t-s)-} \mu^{-n} .
\end{aligned}
$$

Using the spectral expansion of operator $A[\mu \in \sigma(A)]$, we get

$$
G(t-s)= \begin{cases}\sum_{k=0}^{n-1} R_{n}[A(t-s)]^{k} e^{-\left(A+\frac{\alpha}{2} E\right)(t-s)} A^{-n} & \text { if } t>S, \\ T_{n} e^{\left(A-\frac{\alpha}{2} E\right)(t-s)} A^{-n} & \text { if } t<S,\end{cases}
$$

where

$$
R_{n}=2^{(-n)}\left[\frac{2^{(k)}}{(k) !}\right] \text { and } T_{n}=2^{(-n)}
$$

For $v_{0}(t)$, from equation (6) we have

$$
v_{0}(t)=\sum_{k=0}^{n-1}(A t)^{k} e^{-\left(A+\frac{\alpha}{2}\right) t} \phi_{k}
$$

where the vectors $\phi_{k} \in D\left(A^{n+\frac{1}{2}-k}\right), k=\overline{0, n-1}$ :

$$
\begin{aligned}
& \phi_{0}=-v_{1}(0)=-T_{n} \int_{0}^{+\infty} e^{-\left(A-\frac{\alpha}{2} E\right) s} A^{-n} g(s) d s \\
& \phi_{1}=\phi_{0}-A^{-1} v_{1}^{\prime}(0)=-2 T_{n} \int_{0}^{+\infty} e^{-\left(A-\frac{\alpha}{2} E\right) s} A^{-n} g(s) d s \\
& \phi_{2}=\phi_{1}-\frac{1}{2} \phi_{0}-\frac{1}{2} A^{-2} v_{1}^{\prime \prime}(0)=-2 T_{n} \int_{0}^{+\infty} e^{-\left(A-\frac{\alpha}{2} E\right) s} A^{-n} g(s) d s, \\
& \phi_{3}=\frac{1}{6} \phi_{0}-\frac{1}{6} A^{-3} v_{1}^{\prime \prime \prime}(0)-\frac{1}{2} \phi_{1}+\phi_{2}=-\frac{8}{6} T_{n} \int_{0}^{+\infty} e^{-\left(A-\frac{\alpha}{2} E\right) s} A^{-n} g(s) d s,
\end{aligned}
$$

and so on,

$$
\phi_{k}=q_{n} \int_{0}^{+\infty} e^{-\left(A-\frac{\alpha}{2} E\right) s} A^{-n} g(s) d s
$$


Then

$$
\begin{aligned}
& v_{0}(t)=\sum_{k=0}^{n-1} q_{n}(A t)^{k}\left[e^{-\left(A+\frac{\alpha}{2} E\right) t} \times \int_{0}^{+\infty} e^{-\left(A-\frac{\alpha}{2} E\right) s}\left(A^{-n} g(s)\right) d s\right] \\
& q_{n}=-2^{(-n)}\left[\frac{2^{(k)}}{(k) !}\right] .
\end{aligned}
$$

\section{Examples}

(i) If $m=1, n=2$, then

$$
\begin{aligned}
& G(t-s)=\frac{1}{4} \begin{cases}{[E+2(t-s) A] e^{-\left(A+\frac{\alpha}{2} E\right)(t-s)} A^{-2}} & \text { if } t-s>0, \\
e^{\left(A-\frac{\alpha}{2} E\right)(t-s)} A^{-2} & \text { if } t-s<0,\end{cases} \\
& v_{0}(t)=-\frac{1}{4}(E+2 A t)\left[e^{-\left(A+\frac{\alpha}{2} E\right) t} \int_{0}^{+\infty} e^{-\left(A-\frac{\alpha}{2} E\right) s}\left(A^{-2} g(s)\right) d s\right] .
\end{aligned}
$$

(ii) If $m=1, n=3$, then

$$
G(t-s)=\frac{1}{8} \begin{cases}{\left[E+2 A(t-s)+2 A^{2}(t-s)^{2}\right] e^{-\left(A+\frac{\alpha}{2} E\right)(t-s)} A^{-3}} & \text { if } t-s>0, \\ e^{\left(A-\frac{\alpha}{2} E\right)(t-s)} A^{-3} & \text { if } t-s<0,\end{cases}
$$

and

$$
v_{0}(t)=-\frac{1}{8}\left(E+2 A t+2 A^{2} t^{2}\right)\left[e^{-\left(A+\frac{\alpha}{2} E\right) t} \int_{0}^{+\infty} e^{-\left(A-\frac{\alpha}{2} E\right) s}\left(A^{-3} g(s)\right) d s\right]
$$

(iii) If $m=1, n=4$, then

$$
G(t-s)=\frac{1}{16}\left\{\begin{array}{l}
{\left[E+2 A(t-s)+2 A^{2}(t-s)^{2}+\frac{8}{6} A^{3}(t-s)^{3}\right] e^{-\left(A+\frac{\alpha}{2} E\right)(t-s)} A^{-4}} \\
\quad \text { if } t-s>0, \\
e^{\left(A-\frac{\alpha}{2} E\right)(t-s)} A^{-4} \quad \text { if } t-s<0,
\end{array}\right.
$$

and

$$
v_{0}(t)=-\frac{1}{16}\left(E+2 A t+2 A^{2} t^{2}+\frac{8}{6} A^{3} t^{3}\right)\left[e^{-\left(A+\frac{\alpha}{2} E\right) t} \int_{0}^{+\infty} e^{-\left(A-\frac{\alpha}{2} E\right) s}\left(A^{-4} g(s)\right) d s\right]
$$

This case has not been researched so far.

Before we formulate exact conditions on regular solvability of problem (4)-(5), expressed only by its operator coefficients, we must estimate the norms of intermediate derivative operators participating in the second part of equation (4). It follows from Theorem 3 that the norm $\left\|P_{0} u\right\|_{L_{2}\left(R_{+} ; H\right)}$ is equivalent to the norm $\|u\|_{W_{2}^{n+1}\left(R_{+} ; H\right)}$ in the space $W_{2}^{n+1}\left(R_{+} ; H\right)$. Therefore by the norm $\left\|P_{0} u\right\|_{L_{2}\left(R_{+} ; H\right)}$ the theorem on intermediate derivatives is valid as well.

Theorem 4 ([20]) Let the operators $A_{j} A^{-j}, j=\overline{1, n+1}$, be bounded on $H$. Then in the case $A_{j} \neq 0$ the operator $P_{1}$ is bounded from the space $W_{2, \alpha}^{n+1}\left(R_{+} ; H\right)$ to $L_{2, \alpha}\left(R_{+} ; H\right)$. 
Proof Since $u(t) \in w_{2, \alpha}^{n+1}\left(R_{+} ; H\right)$, from the theorem on intermediate derivatives [16, 19] we have

$$
\begin{aligned}
\left\|p_{1} u\right\|_{L_{2, \alpha}\left(R_{+} ; H\right)} & \leq \sum_{j=1}^{n+1}\left\|A_{j} A^{-j}\right\|_{H \rightarrow H}\left\|A^{j} \frac{d^{n-j+1} u}{d t^{n-j+1}}\right\| L_{2, \alpha}\left(R_{+} ; H\right) \\
& \leq \mathrm{const}\|u\|_{w_{2, \alpha}^{n+1}\left(R_{+} ; H\right)} .
\end{aligned}
$$

The theorem is proved.

From Theorems 3 and 4 we get the following lemma.

Lemma 5 Let $A_{j} A^{-j}, j=\overline{1, n+1}$, be bounded operators on $H$. Then in the case $A_{j} \neq 0$ the operator P from the space $W_{2}^{n+1}\left(R_{+} ; H\right)$ to $L_{2}\left(R_{+} ; H\right)$ is bounded [17].

Theorem 6 For functions $u(x) \in W_{2}^{n+1}(R ; H)$, we have the following inequalities:

$$
\left\|A^{n+1-j} \frac{d^{j} u(x)}{d x^{j}}\right\|_{L_{2}(R ; H)} \leq a_{j}\left\|P_{0} u\right\|_{L_{2}(R ; H)}, \quad j=\overline{1, n}
$$

where

$$
a_{j}=\left(\frac{1}{(n+1)^{\frac{n+1}{2}}}\right)\left(j^{j / 2}\right)\left((n-j+1)^{\left(\frac{n-j+1}{2}\right)}\right) \quad(\text { see }[1,4]) .
$$

Let

$$
b_{s}=\left(\frac{1}{(n+1)^{n+1}}\right)\left(s^{s}\right)\left((n-s+1)^{(n-s+1)}\right), \quad s=\overline{1, n} .
$$

Consider the following polynomial operator pencils depending on the real parameter $\beta$ :

$$
P_{j}(\lambda ; \beta ; A)=\left((i \lambda)^{2} E+A^{2}\right)^{n+1}-\beta(i \lambda)^{2 j} A^{(2(n+1)-2 j)}, \quad j=\overline{1, n} .
$$

Let us clarify the study of naturally arising pencils (9). Obviously, for $u(x) \in W_{2}^{n+1}\left(R_{+} ; H\right)$, we have that for estimating $n_{j}, j=\overline{1, n}$, it is necessary to study some properties of pencils.

Theorem 7 Let $\beta \in\left[0, b_{s}^{-1}\right)$. Then the polynomial operator pencils are invertible on the imaginary axis and have the following representations:

$$
P_{j}(\lambda ; \beta ; A)=F_{j}(\lambda ; \beta ; A) F_{j}(-\lambda ; \beta ; A), \quad j=\overline{1, n},
$$

where

$$
F_{j}(\lambda ; \beta ; A)=\prod_{s=1}^{n+1}\left(\lambda E-\omega_{j, s}(\beta) A\right) \equiv \sum_{m=0}^{n+1} \alpha_{m, j}(\beta) \lambda^{(n-m)} A^{m},
$$

$\operatorname{Re} \omega_{j, s}(\beta)<0, s=\overline{1, n+1}$, and the numbers $\alpha_{m, j}(\beta)>0, m=\overline{0, n+1}$;

$\alpha_{v, j}(\beta)>0, v=\overline{0, n+1}$, satisfy the following systems of equations:

$$
P_{j}(\lambda ; \beta ; A)=\left((i \lambda)^{2} E+A^{2}\right)^{n+1}-\beta(i \lambda)^{2 j} A^{(2(n+1)-2 j)}
$$




$$
=\sum_{m=0}^{n+1}\left[\sum_{\nu=0}^{n+1} \alpha_{m, j}(\beta) \alpha_{\nu, j}(\beta) \lambda^{(n-m+1)}(-\lambda)^{(n-v+1)} A^{m+\nu}\right] .
$$

\section{Examples}

(i) if $m=1$ and $n=2$, then the following systems of equations are satisfied:

(1) for $j=1$,

$$
\left\{\begin{array}{l}
-2 \alpha_{2,1}(\beta)+\alpha_{1,1}^{2}(\beta)-3=0 \\
2 \alpha_{1,1}(\beta)-\alpha_{2,1}^{2}(\beta)+3=\beta
\end{array}\right.
$$

(2) for $j=2$,

$$
\left\{\begin{array}{l}
-2 \alpha_{2,2}(\beta)+\alpha_{1,2}^{2}(\beta)-3=-\beta \\
2 \alpha_{1,2}(\beta)-\alpha_{2,2}^{2}(\beta)+3=0
\end{array}\right.
$$

(ii) if $m=1$ and $n=3$, then the following systems of equations are satisfied:

(1) for $j=1$,

$$
\left\{\begin{array}{l}
2 \alpha_{2,1}(\beta)-\alpha_{1,1}^{2}(\beta)+4=0, \\
\alpha_{2,1}^{2}(\beta)-2 \alpha_{1,1}(\beta) \alpha_{3,1}(\beta)-4=0 \\
2 \alpha_{2,1}(\beta)-\alpha_{3,1}^{2}(\beta)+4=\beta
\end{array}\right.
$$

(2) for $j=2$,

$$
\left\{\begin{array}{l}
2 \alpha_{2,2}(\beta)-\alpha_{1,2}^{2}(\beta)+4=0 \\
\alpha_{2,2}^{2}(\beta)-2 \alpha_{1,2}(\beta) \alpha_{3,2}(\beta)-4=-\beta \\
2 \alpha_{2,2}(\beta)-\alpha_{3,2}^{2}(\beta)+4=0
\end{array}\right.
$$

(3) for $j=3$,

$$
\left\{\begin{array}{l}
2 \alpha_{2,3}(\beta)-\alpha_{1,3}^{2}(\beta)+4=\beta, \\
\alpha_{2,3}^{2}(\beta)-2 \alpha_{1,3}(\beta) \alpha_{3,3}(\beta)-4=0, \\
2 \alpha_{2,3}(\beta)-\alpha_{3,3}^{2}(\beta)+4=0
\end{array}\right.
$$

(iii) if $m=1$ and $n=4$, then:

(1) for $j=1$,

$$
\left\{\begin{array}{l}
-2 \alpha_{2,1}(\beta)+\alpha_{1,1}^{2}(\beta)-5=0 \\
2 \alpha_{1,1}(\beta) \alpha_{3,1}(\beta)-2 \alpha_{4,1}(\beta)-\alpha_{2,1}^{2}(\beta)+10=0 \\
2 \alpha_{1,1}(\beta)-2 \alpha_{2,1}(\beta) \alpha_{4,1}(\beta)+\alpha_{3,1}^{2}(\beta)-10=0 \\
2 \alpha_{3,1}(\beta)-\alpha_{4,1}^{2}(\beta)+5=\beta
\end{array}\right.
$$


(2) for $j=2$,

$$
\left\{\begin{array}{l}
-2 \alpha_{2,2}(\beta)+\alpha_{1,2}^{2}(\beta)-5=0 \\
2 \alpha_{1,2}(\beta) \alpha_{3,2}(\beta)-2 \alpha_{4,2}(\beta)-\alpha_{2,2}^{2}(\beta)+10=0 \\
2 \alpha_{1,2}(\beta)-2 \alpha_{2,2}(\beta) \alpha_{4,2}(\beta)+\alpha_{3,2}^{2}(\beta)-10=-\beta \\
2 \alpha_{3,2}(\beta)-\alpha_{4,2}^{2}(\beta)+5=0
\end{array}\right.
$$

(3) for $j=3$,

$$
\left\{\begin{array}{l}
-2 \alpha_{2,3}(\beta)+\alpha_{1,3}^{2}(\beta)-5=0 \\
2 \alpha_{1,3}(\beta) \alpha_{3,3}(\beta)-2 \alpha_{4,3}(\beta)-\alpha_{2,3}^{2}(\beta)+10=\beta \\
2 \alpha_{1,3}(\beta)-2 \alpha_{2,3}(\beta) \alpha_{4,3}(\beta)+\alpha_{3,3}^{2}(\beta)-10=0 \\
2 \alpha_{3,3}(\beta)-\alpha_{4,3}^{2}(\beta)+5=0
\end{array}\right.
$$

(4) for $j=4$,

$$
\left\{\begin{array}{l}
2 \alpha_{2,4}(\beta)+\alpha_{1,4}^{2}(\beta)-5=-\beta \\
2 \alpha_{1,4}(\beta) \alpha_{3,4}(\beta)-2 \alpha_{4,4}(\beta)-\alpha_{2,4}^{2}(\beta)+10=0 \\
2 \alpha_{1,4}(\beta)-2 \alpha_{2,4}(\beta) \alpha_{4,4}(\beta)+\alpha_{3,4}^{2}(\beta)-10=0 \\
2 \alpha_{3,4}(\beta)-\alpha_{4,4}^{2}(\beta)+5=0
\end{array}\right.
$$

This case has not been researched yet.

Theorem 8 Let $\beta \in\left[0, b_{s}^{-1}\right)$. Then for any $u(t) \in W_{2}^{n+1}\left(R_{+} ; H\right)$, the following relation holds:

$$
\left\|F_{j}\left(\frac{d}{d t} ; \beta ; A\right) u\right\|_{L_{2}\left(R_{+} ; H\right)}^{2}=\left\|P_{0} u\right\|_{L_{2}\left(R_{+} ; H\right)}^{2}-\beta\left\|A^{n-j+1} \frac{d^{j}}{d t^{j}} u\right\|_{L_{2}\left(R_{+} ; H\right)}^{2}, \quad j=\overline{1, n},
$$

on the values of the numbers $n_{j}, j=\overline{1, n}$.

Theorem 3 implies that the norms $W_{2}^{n+1}\left(R_{+} ; H\right)$ and $\left\|P_{0} u\right\|_{L_{2}\left(R_{+} ; H\right)}$ are equivalent on $W_{2}^{n+1}\left(R_{+} ; H\right)$. Then it follows from the theorem of intermediate derivatives that the following numbers are finite:

$$
n_{j}=\sup _{0 \neq u \in W_{2}^{n+1}\left(R_{+} ; H\right)} \frac{\left\|A^{n-j+1} \frac{d^{j}}{d \dot{j}} u\right\|_{L_{2}\left(R_{+} ; H\right)}}{\left\|P_{0} u\right\|_{L_{2}\left(R_{+} ; H\right)}}, \quad j=\overline{1, n}
$$

Now let us calculate $n_{j}$.

Lemma $9([18]) n_{j}=a_{j}, j=\overline{1, n}$.

Corollary 10 Let $\gamma \in \sigma(A), \gamma \geq \gamma_{0}\left(\gamma_{0}>0\right)$, and $|\alpha|<2 \gamma_{0}, \xi \in R$ Then

$$
\left\|A^{n+1} P_{0}^{-1}\left(i \xi+\frac{\alpha}{2}, A\right)\right\| \leq c_{j}\left(\alpha, \gamma_{0}\right), \quad j=n+1
$$


where

$$
c_{j}\left(\alpha, \gamma_{0}\right)=\frac{\left(\gamma_{0}\right)^{n+1}}{\left(\gamma_{0}^{2}-\frac{\alpha^{2}}{4}\right)\left(\gamma_{0}+\frac{\alpha}{2}\right)^{n-1}} .
$$

Proof Since $A$ is a self-adjoint positive operator, from the spectral theory we have

$$
\begin{aligned}
& \left\|A^{n+1} p_{0}^{-1}\left(i \xi+\frac{\alpha}{2}, A\right)\right\| \\
& =\sup _{\gamma \in \sigma(A)}\left|\gamma^{n+1}\left[-\left(i \xi+\frac{\alpha}{2}\right)+\gamma\right]^{-1}\left[i \xi+\frac{\alpha}{2}+\gamma\right]^{-n}\right| \\
& =\sup _{\gamma \in \sigma(A)}\left|\gamma^{n+1}\left[-\left(i \xi+\frac{\alpha}{2}\right)^{2}+\gamma^{2}\right]^{-1}\left[i \xi+\frac{\alpha}{2}+\gamma\right]^{-n+1}\right| \\
& \leq \sup _{\gamma \in \sigma(A)} \frac{(\gamma)^{n+1}}{\left(\xi^{2}+\gamma^{2}-\frac{\alpha^{2}}{4}\right)\left[\left(\gamma+\frac{\alpha}{2}\right)^{2}+\xi^{2}\right]^{\frac{n-1}{2}}} \\
& \leq \sup _{\gamma \in \sigma(A)} \frac{(\gamma)^{n+1}}{\left(\gamma^{2}-\frac{\alpha^{2}}{4}\right)\left(\gamma+\frac{\alpha}{2}\right)^{n-1}} \\
& \leq \frac{\left(\gamma_{0}\right)^{n+1}}{\left(\gamma_{0}^{2}-\frac{\alpha^{2}}{4}\right)\left(\gamma_{0}+\frac{\alpha}{2}\right)^{n-1}}=c_{j}\left(\alpha, \gamma_{0}\right), \quad j=n+1 .
\end{aligned}
$$

Theorem 11 ([4]) Let $-2 \gamma_{0}<\alpha<2 \gamma_{0}$. Then for any $u(t) \in W_{2, \alpha}^{n+1}\left(R_{+} ; H\right)$, we have the inequalities

$$
\left\|A^{j} \frac{d^{n-j+1} u(t)}{d t^{n-j+1}}\right\|_{L_{2}\left(R_{+} ; H\right)} \leq b\left(\alpha, \gamma_{0}\right) c_{j}\left(\alpha, \gamma_{0}\right)\left\|P_{0} u(t)\right\|_{L_{2, \alpha}\left(R_{+} ; H\right)}, \quad j=\overline{1, n+1}
$$

where

$$
c_{j}\left(\alpha, \gamma_{0}\right)=\left(\frac{2 \gamma_{0}}{2 \gamma_{0}+\alpha}\right)^{j-1}\left[1+\frac{4 \gamma_{0}\left|\gamma_{0}+\alpha\right|}{\left(2 \gamma_{0}+\alpha\right)^{2}}\right]^{\frac{n-j}{2}}, \quad j=\overline{1, n}
$$

From Corollary 10 we obtain

$$
c_{j}\left(\alpha, \gamma_{0}\right)=\frac{\left(\gamma_{0}\right)^{n+1}}{\left(\gamma_{0}^{2}-\frac{\alpha^{2}}{4}\right)\left(\gamma_{0}+\frac{\alpha}{2}\right)^{n-1}}, \quad j=n+1
$$

and

$$
b\left(\alpha, \gamma_{0}\right)= \begin{cases}\frac{\gamma_{0}}{\sqrt{2\left(2 \gamma_{0}^{2}-\alpha^{2}\right)}} & \text { if } 0 \leq \frac{\alpha^{2}}{4 \gamma_{0}^{2}}<\frac{1}{3}, \\ \frac{2 \gamma_{0}|\alpha|}{4 \gamma_{0}^{2}-\alpha^{2}} & \text { if } \frac{1}{3} \leq \frac{\alpha^{2}}{4 \gamma_{0}^{2}}<1 .\end{cases}
$$

Now we introduce the following specific cases at certain values of $n$.

Case (i) $m=1, n=1$. Then we have an initial-boundary value problem of a second-order operator-differential equation with multiple characteristics with

$$
c_{1}\left(\alpha, \gamma_{0}\right)=1 \text {, }
$$




$$
C_{2}\left(\alpha, \gamma_{0}\right)=\frac{\left(\gamma_{0}\right)^{2}}{\left(\gamma_{0}^{2}-\frac{\alpha^{2}}{4}\right)},
$$

and

$$
b\left(\alpha, \gamma_{0}\right)= \begin{cases}\frac{\gamma_{0}}{\sqrt{2\left(2 \gamma_{0}^{2}-\alpha^{2}\right)}} & \text { if } 0 \leq \frac{\alpha^{2}}{4 \gamma_{0}^{2}}<\frac{1}{3}, \\ \frac{2 \gamma_{0}|\alpha|}{4 \gamma_{0}^{2}-\alpha^{2}} & \text { if } \frac{1}{3} \leq \frac{\alpha^{2}}{4 \gamma_{0}^{2}}<1 .\end{cases}
$$

Case (ii) $m=1, n=2$. Then we have an initial-boundary value problem of a third-order operator-differential equation with multiple characteristics with

$$
\begin{aligned}
& c_{1}\left(\alpha, \gamma_{0}\right)=\left[1+\frac{4 \gamma_{0}\left|\gamma_{0}+\alpha\right|}{\left(2 \gamma_{0}+\alpha\right)^{2}}\right]^{\frac{1}{2}}, \quad c_{2}\left(\alpha, \gamma_{0}\right)=\frac{2 \gamma_{0}}{2 \gamma_{0}+\alpha}, \\
& c_{3}\left(\alpha, \gamma_{0}\right)=\frac{\left(\gamma_{0}\right)^{3}}{\left(\gamma_{0}^{2}-\frac{\alpha^{2}}{4}\right)\left(\gamma_{0}+\frac{\alpha}{2}\right)},
\end{aligned}
$$

and

$$
b\left(\alpha, \gamma_{0}\right)= \begin{cases}\frac{\gamma_{0}}{\sqrt{2\left(2 \gamma_{0}^{2}-\alpha^{2}\right)}} & \text { if } 0 \leq \frac{\alpha^{2}}{4 \gamma_{0}^{2}}<\frac{1}{3}, \\ \frac{2 \gamma_{0}|\alpha|}{4 \gamma_{0}^{2}-\alpha^{2}} & \text { if } \frac{1}{3} \leq \frac{\alpha^{2}}{4 \gamma_{0}^{2}}<1 .\end{cases}
$$

Case (iii) $m=1, n=3$. Then we have an initial-boundary value problem of a fourth-order operator-differential equation with multiple characteristics with

$$
\begin{aligned}
& c_{1}\left(\alpha, \gamma_{0}\right)=\left[1+\frac{4 \gamma_{0}\left|\gamma_{0}+\alpha\right|}{\left(2 \gamma_{0}+\alpha\right)^{2}}\right], \\
& c_{2}\left(\alpha, \gamma_{0}\right)=\left(\frac{2 \gamma_{0}}{2 \gamma_{0}+\alpha}\right)\left[1+\frac{4 \gamma_{0}\left|\gamma_{0}+\alpha\right|}{\left(2 \gamma_{0}+\alpha\right)^{2}}\right]^{\frac{1}{2}}, \\
& c_{3}\left(\alpha, \gamma_{0}\right)=\left(\frac{2 \gamma_{0}}{2 \gamma_{0}+\alpha}\right), \\
& c_{4}\left(\alpha, \gamma_{0}\right)=\frac{\left(\gamma_{0}\right)^{4}}{\left(\gamma_{0}^{2}-\frac{\alpha^{2}}{4}\right)\left(\gamma_{0}+\frac{\alpha}{2}\right)^{2}},
\end{aligned}
$$

and

$$
b\left(\alpha, \gamma_{0}\right)= \begin{cases}\frac{\gamma_{0}}{\sqrt{2\left(2 \gamma_{0}^{2}-\alpha^{2}\right)}} & \text { if } 0 \leq \frac{\alpha^{2}}{4 \gamma_{0}^{2}}<\frac{1}{3}, \\ \frac{2 \gamma_{0}|\alpha|}{4 \gamma_{0}^{2}-\alpha^{2}} & \text { if } \frac{1}{3} \leq \frac{\alpha^{2}}{4 \gamma_{0}^{2}}<1 .\end{cases}
$$

Theorem 12 Let $A=A^{*}, A^{*} \geq \gamma_{0} E\left(-2 \gamma_{0}<\alpha<2 \gamma_{0}, \gamma_{0}>0\right)$, and let the operators $A_{j} A^{-j}(j=$ $\overline{1, n+1}$ ) be bounded in $H$ and satisfy the inequality

$$
\sum_{j=1}^{n+1} c_{j}\left(\alpha, \gamma_{0}\right) b\left(\alpha, \gamma_{0}\right)\left\|A_{j} A^{-j}\right\|_{H \rightarrow H}<1,
$$

where the numbers $b\left(\alpha, \gamma_{0}\right)$ and $c_{j}\left(\alpha, \gamma_{0}\right), j=\overline{1, n+1}$, are calculated in Theorem 11 . Then the initial-boundary value problem (4)-(5) is regularly solvable [1]. 
Proof Let $f(t) \in L_{2, \alpha}\left(R_{+} ; H\right)$ and $u(t) \in W_{2, \alpha}^{n+1}\left(R_{+} ; H\right)$. By Theorem 3 there exists a bounded inverse operator for $P_{0}$ that acts from $L_{2, \alpha}\left(R_{+} ; H\right)$ to $W_{2, \alpha}^{n+1}\left(R_{+} ; H\right)$. Then after substituting $P_{0} u(t)=w(t)$ into equation (4), the latter can be written as $\left(E+\left(P-P_{0}\right) P_{0}^{-1} w(t)=f(t)\right.$. Now to show the existence of a solution, let us check that

$$
\left\|P_{1} P_{0}^{-1}\right\|_{L_{2, \alpha}\left(R_{+} ; H\right) \rightarrow L_{2, \alpha}\left(R_{+} ; H\right)}<1 .
$$

By Theorem 4 we have:

$$
\begin{aligned}
& \left\|P_{1} P_{0}^{-1} w(t)\right\|_{L_{2, \alpha}\left(R_{+} ; H\right)} \\
& =\left\|P_{1} u(t)\right\|_{L_{2}\left(R_{+} ; H\right)} \leq \sum_{j=1}^{n+1}\left\|A_{j} \frac{d^{n-j+1} u(t)}{d t^{n-j+1}}\right\|_{L_{2, \alpha}\left(R_{+} ; H\right)} \\
& \leq \sum_{j=1}^{n+1}\left\|A_{j} A^{-j}\right\|_{H \rightarrow H}\left\|A^{j} \frac{d^{n-j+1} u(t)}{d t^{n-j+1}}\right\|_{L_{2, \alpha}\left(R_{+} ; H\right)} \\
& \leq \sum_{j=1}^{n+1}\left\|A_{j} A^{-j}\right\|_{H \rightarrow H} b\left(\alpha, \gamma_{0}\right) c_{j}\left(\alpha, \gamma_{0}\right)\left\|P_{0} u(t)\right\|_{L_{2, \alpha}\left(R_{+} ; H\right)} \\
& \quad=\sum_{j=1}^{n+1} b\left(\alpha, \gamma_{0}\right) c_{j}\left(\alpha, \gamma_{0}\right)\left\|A_{j} A^{-j}\right\|_{H \rightarrow H}\|w(t)\|_{L_{2, \alpha}\left(R_{+} ; H\right)}
\end{aligned}
$$

Consequently,

$$
\left\|P_{1} P_{0}^{-1}\right\|_{L_{2, \alpha}\left(R_{+} ; H\right) \rightarrow L_{2, \alpha}\left(R_{+} ; H\right)} \leq \sum_{j=1}^{n+1} b\left(\alpha, \gamma_{0}\right) c_{j}\left(\alpha, \gamma_{0}\right)\left\|A_{j} A^{-j}\right\|_{H \rightarrow H}<1
$$

Thus the operator $\left(E+\left(P-P_{0}\right) P_{0}^{-1}\right)$ is invertible in $L_{2, \alpha}\left(R_{+} ; H\right)$, and therefore $u(t)$ can be determined as $u(t)=P_{0}^{-1}\left(E+\left(P-P_{0}\right) P_{0}^{-1}\right)^{-1} f(t)$.

Moreover,

$$
\begin{aligned}
& \|u(t)\|_{W_{2, \alpha}^{n+1}\left(R_{+} ; H\right)} \\
& \leq\left\|P_{0}^{-1}\right\|_{L 2, \alpha\left(R_{+} ; H\right) \rightarrow W_{2, \alpha}^{n+1}\left(R_{+} ; H\right)} \\
& \quad \times\left\|\left(\left(E+\left(P-P_{0}\right) P_{0}^{-1}\right)\right)^{-1}\right\|_{L 2, \alpha\left(R_{+} ; H\right) \rightarrow L 2, \alpha\left(R_{+} ; H\right)}\|f(t)\|_{L 2, \alpha\left(R_{+} ; H\right)} \\
& \leq \text { const }\|f(t)\|_{L 2, \alpha\left(R_{+} ; H\right)} .
\end{aligned}
$$

\section{Example}

Using the results on the solvability of initial-boundary value problem (4)-(5), we introduce the following problem as an application example on the half-strip $R_{+} \times[0 ; \pi][21]$ :

$$
\begin{aligned}
& \left(-\frac{\partial}{\partial t}-\frac{\partial^{2}}{\partial x^{2}}\right)\left(\frac{\partial}{\partial t}-\frac{\partial^{2}}{\partial x^{2}}\right)^{n} u(t, x)+\sum_{j=1}^{n+1} r_{j}(x) \frac{\partial^{n+j+1} u(t, x)}{\partial t^{n-j+1} \partial x^{2 j}}=f(t, x), \\
& \frac{\partial^{s} u(x, 0)}{\partial t^{s}}=0, \quad s=\overline{0, n-1},
\end{aligned}
$$




$$
\frac{\partial^{2 k} u(t, 0)}{\partial x^{2 k}}=\frac{\partial^{2 k} u(t, \pi)}{\partial x^{2 k}}=0, \quad k=\overline{0, n}
$$

where $r_{j}(x), j=\overline{1, n+1}$, are bounded functions on $[0, \pi], f(t, x) \in L_{2}\left(R_{+} ; L_{2}[0, \pi]\right)$. Note that problem (10)-(12) is a particular case of boundary value problem (4)-(5), where $A_{j}=r_{j}(x) \frac{\partial^{2 j}}{\partial x^{2 j}}, j=\overline{1, n+1}$, is the operator is defined on $H=L_{2, \alpha}[0, \pi]$ by $A u=-\frac{d^{2} u}{d x^{2}}$ and the conditions $\left.u\right|_{x=0}=\left.u\right|_{x-\pi}=0$. Then problem (10)-(12) has a unique solution in the space $W_{t, x, 2}^{n+1,2(n+1)}\left(R_{+} ; L_{2}[0 ; \pi]\right)$.

In the case $\gamma_{0}=1$, applying Theorem 12 , in the space $W_{t, x, 2, \alpha}^{n+1,2(n+1)}\left(R_{+} ; L_{2}[0 ; \pi]\right)$ with $-2<$ $\alpha<2$, we get the inequality

$$
\left[\sum_{j=1}^{n+1} c_{j}(\alpha, 1) b(\alpha, 1) \sup _{x \in[0, \pi]}\left|r_{j}(x)\right|\right]<1 .
$$

Hence the mixed partial differential equation has a unique solution.

\section{Conclusion}

In a Sobolev-type space with exponential weight, we found a solution of the initialboundary value problem of $(n+1)$ th-order operator-differential equation in the case that the second part equals zero. We obtained definite conditions for problem (4)-(5) to be regularly solvable; these conditions rely on the operator coefficients, the lower bound of the spectrum, and the weight exponent. The estimates of the norms of the intermediate derivatives of the differential operators in the substantial part of the given equation are provided and, in a similar way, for the second part of the above-mentioned equation.

We used the results of this paper to establish an application example of the initialboundary value problem (10)-(12) for mixed partial differential equations. The wellposedness of problem (4)-(5) is also proved using the polynomial operator pencils.

Acknowledgements

We would like to thank the anonymous referees for their constructive and helpful comments, which improved the quality of this paper.

Funding

Not applicable.

Availability of data and materials

Not applicable.

Competing interests

The authors declare that they have no competing interests.

Authors' contributions

The authors contributed equally to this work. All authors read and approved the final manuscript.

Author details

${ }^{1}$ Ain Shams University, Cairo, Egypt. ${ }^{2}$ Helwan University, Cairo, Egypt. ${ }^{3}$ Egyptian Russian University, Cairo, Egypt.

\section{Publisher's Note}

Springer Nature remains neutral with regard to jurisdictional claims in published maps and institutional affiliations.

Received: 10 November 2019 Accepted: 19 February 2020 Published online: 05 March 2020

References

1. Ahmed, A.B.I., Labeeb, M.A.: Solvability of a class of operator-differential equations of third order with complicated characteristic on the whole real axis. Open Access Libr. J. 5, 6 (2018) 
2. Amirova, E.G., Karaaslan, M.D.: On solvability of a boundary value problem for second order operator differential equations in the space of smooth vector-functions. In: Transactions of NAS of Azerbaijan, vol. XXXV, pp. 24-30 (2015)

3. Amirova, L.I.: On the solvability of boundary value problem for a class of second order operator differential equations. In: Proceedings of IMM of Azerbaijan AS, vol. XIXIX, pp. 16-20 (2000)

4. Faried, N., Rashed, L., Ahmed, A.B.I., Labeeb, M.A.: Solvability of initial-boundary value problem of a multiple characteristic fifth-order operator-differential equation. J. Egypt. Math. Soc. 27, 1-7 (2019)

5. Gamidov, E.G.: On a boundary value problem for second order operator differential equations in space of smooth vector functions. In: Transactions of NAS of Azerbaijan, vol. XXXIII, pp. 72-84 (2013)

6. Gamidov, E.G., Karaaslan, M.D.: On solvability of a boundary value problem for second order operator differential equations in the space of smooth vector functions. In: Transactions of NAS of Azerbaijan, vol. XXXV, pp. 24-30 (2015)

7. Gasymov, A.A.: On solvability of a class of complicated characteristic operator differential equations of fourth order. In: Transactions of NAS of Azerbaijan, vol. 28, pp. 49-54 (2007)

8. Gasymov, M.G.: On multiple completeness of a part of eigen and associated vectors of polynomial operator pencils, Izv. AS Arm. SSR, Ser. Mathematics. 6, 131-147 (1971)

9. Gasymov, M.G.: To theory of polynomial operator pencils. DAN SSSR. 149, 747-750 (1971)

10. Gasymov, M.G., Mirzoev, S.S.: On solvability of boundary value problems for second order operator-differential equations of elliptic type. Differ. Uravn. 28, 651-661 (1992)

11. Gorbachuk, V.I., Gorbachuk, M.L.: Boundary Value Problems for Differential-Operator Equations, vol. 284. Naukova Dumka, Kiev (1984) (Russian)

12. Humbataliev, R.Z:: On regular solvability of boundary value problems in weight space. Int. J. Math. Anal. 1, 1209-1216 (2007)

13. Komornik, V.: Lectures on Functional Analysis and the Lebesgue Integral, vol. 2. Springer, London (2016)

14. Krein, S.G.: Linear Equations in Banach Spaces, vol. 112. Birkhäuser, Boston (1982)

15. Kreyszig, E.: Introductory Functional Analysis with Applications. Wiley, New York (1989)

16. Krylov, N.V.: Lectures on elliptic and parabolic equations in Sobolev spaces. Am. Math. Soc. 96, 357 (2008)

17. Labeeb, M.A., Ahmed, A.B.I., Rashed, L.: Conditional solvability of the boundary value problem of a self-adjoint operator-differential equations in a Sobolev-type space. Int. J. Appl. Math. 32, 469-478 (2019)

18. Lachinova, F.S.: Solvability of a class of parabolic operator-differentia equations of third order. In: Proceedings of IMM of NAS of Azerbaijan, vol. XXXIX, pp. 77-86 (2013)

19. Lions, L.J., Majenes, E.: Non-homogeneous Boundary Value Problems and Their Applications. Springer, Berlin (1972)

20. Lusternik, L.A., Sobolev, V.J.: Elements of Functional Analysis. India, 376 (1979). (Russian)

21. Mamedov, M.M.: Solvability and completeness of solutions of parabolic differential operator equations. Mat. Stud. 36, $77-85(2011)$

22. Mirzoev, S.S.: On solvability of boundary value problems for operator differential equations of the second order in spaces with weight. In: Linear Operators and Their Applications, pp. 46-49. ASU, Baku (1989) (Russian)

23. Mirzoev, S.S., Karaaslan, M.D., Gumbataliev, R.Z.: To the theory of operator differential equations of the second order. Dokl. Math. 88, 741-743 (2013)

24. Mirzoev, S.S., Salimov, M.Y.: On the solvability of the boundary value problem for second order equations in Hilbert space with an operator coefficient in the boundary condition. Mat. Zametki 91, 861-869 (2012)

25. Pilipchuk, V.N.: On essentially nonlinear dynamics of arches and rings. J. Appl. Math. Mech. 46, 461-466 (1982) (Russian)

26. Teters, G.A.: Complex Loading and Stability of the Covers from Polymeric Materials. Zinatne Press, Latvia (1969) (Russian)

27. Yakubov, S., Yakubov, Y.: Differential-operator equations: ordinary and partial differential equations. In: Pure and Applied Mathematics. Elm, USA (1999)

\section{Submit your manuscript to a SpringerOpen ${ }^{\circ}$ journal and benefit from:}

- Convenient online submission

- Rigorous peer review

- Open access: articles freely available online

- High visibility within the field

- Retaining the copyright to your article

Submit your next manuscript at $\boldsymbol{~ s p r i n g e r o p e n . c o m ~}$ 